\title{
Benchmarking of next and third generation sequencing technologies and their associated algorithms for de novo genome assembly
}

\author{
MARIOS GAVRIELATOS ${ }^{1,2}$, KONSTANTINOS KYRIAKIDIS ${ }^{3,4}$, \\ DEMETRIOS A. SPANDIDOS ${ }^{5}$ and IOANNIS MICHALOPOULOS ${ }^{1}$
}

\author{
${ }^{1}$ Centre of Systems Biology, Biomedical Research Foundation, Academy of Athens, 11527 Athens; \\ ${ }^{2}$ Department of Cell Biology and Biophysics, Faculty of Biology, University of Athens, 15701 Athens; \\ ${ }^{3}$ School of Pharmacy, Aristotle University of Thessaloniki (AUTh), 54124 Thessaloniki; \\ ${ }^{4}$ Genomics and Epigenomics Translational Research (GENeTres), Centre for Interdisciplinary Research and Innovation, \\ 57001 Thessaloniki; ${ }^{5}$ Laboratory of Clinical Virology, Medical School, University of Crete, 71003 Heraklion, Greece
}

Received November 4, 2020; Accepted January 21, 2021

DOI: $10.3892 / \mathrm{mmr} .2021 .11890$

\begin{abstract}
Genome assemblers are computational tools for de novo genome assembly, based on a plenitude of primary sequencing data. The quality of genome assemblies is estimated by their contiguity and the occurrences of misassemblies (duplications, deletions, translocations or inversions). The rapid development of sequencing technologies has enabled the rise of novel de novo genome assembly strategies. The ultimate goal of such strategies is to utilise the features of each sequencing platform in order to address the existing weaknesses of each sequencing type and compose a complete and correct genome map. In the present study, the hybrid strategy, which is based on Illumina short paired-end reads and Nanopore long reads, was benchmarked using MaSuRCA and Wengan assemblers. Moreover, the long-read assembly strategy, which is based on Nanopore reads, was benchmarked using Canu or PacBio HiFi reads were benchmarked using Hifiasm and HiCanu. The assemblies were performed on a computational cluster with limited computational resources. Their outputs were evaluated in terms of accuracy and computational performance. PacBio $\mathrm{HiFi}$ assembly strategy outperforms the other ones, while Hi-C scaffolding, which is based on chromatin 3D structure, is required in order to increase continuity, accuracy and completeness when large and complex genomes, such as the human one, are assembled. The use of Hi-C data is also necessary while using the hybrid assembly strategy. The results revealed that $\mathrm{HiFi}$ sequencing enabled the rise of novel algorithms which require less genome coverage than that of the other strategies
\end{abstract}

Correspondence to: Dr Ioannis Michalopoulos, Centre of Systems Biology, Biomedical Research Foundation, Academy of Athens, 4 Soranou Efessiou, 11527 Athens, Greece

E-mail: imichalop@bioacademy.gr

Key words: de novo genome assembly, next generation sequencing, third generation sequencing, genomics, benchmarking, bioinformatics making the assembly a less computationally demanding task. Taken together, these developments may lead to the democratisation of genome assembly projects which are now approachable by smaller labs with limited technical and financial resources.

\section{Introduction}

The first human genome draft (1) was based on Sanger sequencing technology (2), cost $\$ 2.7$ billion and lasted over a period of 10 years (3). In comparison, the sequencing of the human genome ( $\sim 3 \mathrm{Gbp}$ haploid genome size) in a next generation sequencing (NGS) platform where millions of reads are efficiently mapped to the reference genome, currently costs $<\$ 1,000$ and it can be performed in $<2$ days (4). Short-read de novo genome assemblers have difficulty to produce large and reliable contigs, particularly in low complexity regions such as centromeres, telomeres and other repetitive regions $(5,6)$. To address this issue, third generation sequencing (7) technologies have been developed. Nanopore (https://nanoporetech.com/) $(8,9)$ and PacBio (https://www.pacb.com/) (10) sequencing platforms were launched around 2010. Third generation sequencers are sequencing single-molecules in real-time (10) without the need of PCR amplification and thus, avoid PCR bias $(11,12)$. The main drawback of long reads is lower accuracy compared to Illumina short-reads: Typical Nanopore and PacBio Sequel I long-reads have an average accuracy of $90 \%$ (13) compared to $99.9 \%$ of typical Illumina short-reads (4). As a consequence, assemblies produced only by long-reads were more contiguous, but they also contained more errors, which made genome annotation, variant calling and other genome analyses, challenging tasks $(6,12)$.

By following the hybrid assembly strategy $(14,15)$, the advantages of the two generations are combined, incorporating the information contained in the two read types, overcoming their drawbacks. Recent advantages in long-read sequencing by PacBio have shown very promising results: Sequel System II was released in 2019 with an upgraded SMRT flow cell that was first introduced in 2013 (16), which was able to increase the 
sequencing yield up to 8-fold. However, the greatest breakthrough was the advance of circular consensus sequencing (CCS) (17) which sequences the same circular DNA molecule 10 times, to produce a highly accurate $(99.9 \%)$ high-fidelity ( $\mathrm{HiFi})$ consensus read, while increasing unique molecular yield and insert size (up to $25 \mathrm{Kbp}$ ). At the same time, recent advances in Nanopore's base identification algorithm, Bonito (https://github.com/nanoporetech/bonito) (18), have led to greater than $97 \%$ base accuracy.

Usually, the primary genome assembly is very fragmented and some contigs are misassembled. For this reason, the completion of the assembly requires the construction of scaffolds (19). To this end, Hi-C sequencing method provides chromosomal conformation information necessary to assemble chromosome-level scaffolds. The general principle of this method is based on the proximity and contacts of chromosomal regions in the cell nucleus. The frequency of contacts is higher between regions of the same chromosome; thus, different chromosomes can be distinguished during the assembly (20). The result of this method is a collection of pairs of reads of chimeric fragments that can be mapped to the assembly, joining very remote areas.

Using the recent sequencing and scaffolding technologies, it is now possible to construct new reference genomes and finish the assembly of existing ones, by closing gaps in the centromeres, telomeres and other low complexity regions. For this reason, new projects have been launched and new consortia have been formed (21-23). The telomere to telomere (T2T) consortium (https://sites.google.com/ucsc.edu/t2tworkinggroup/) $(24,25)$ aims to finish the entire human genome by producing chromosomes without gaps. Almost two decades after the first draft of the human genome by the International Human Genome Sequencing Consortium, T2T published a completed human genome with the exception of five known gaps withing the rDNA arrays (https://genomeinformatics.github.io/CHM13v1/).

The development of sequencing technologies and assembly and scaffolding algorithms, as well as the sharp increase of publicly available data (https://www.ncbi.nlm. nih.gov/genbank/statistics/), democratised de novo genome assembly projects by making them more approachable to smaller labs. The present study aimed to compare genome assembly pipelines, which use different assembly strategies, evaluating them in terms of accuracy, speed and computational power needed. Finally, the need for scaffold construction, incorporating $\mathrm{Hi}-\mathrm{C}$ sequencing data was also evaluated.

\section{Materials and methods}

Data acquisition and experimental overview. Primary sequencing data were downloaded from 3 organisms, Drosophila virilis, Drosophila melanogaster and Homo sapiens (Table I). Some FASTQ files were subsampled using Reformat tool from BBtools (https://sourceforge.net/projects/bbmap/). Following the hybrid assembly strategy, using short paired-end Illumina reads in combination with long Nanopore reads, the low complexity genome of Drosophila virilis and the high complexity genome of Homo sapiens were constructed, downloading read data from the European Nucleotide Archive (ENA) (26) and the T2T Consortium, respectively. Drosophila melanogaster genome was assembled following the long-read assembly strategy using only HiFi reads retrieved from ENA. Finally, Hi-C reads were used to create the scaffolds of our assemblies. It is important to note that the sequencing data used to assemble Homo sapiens genome, derives from CHM13hTERT, which is a female haploid cell line; thus, there will be no Y chromosome in the final assemblies. The experiments were performed on the Biomedical Research Foundation, Academy of Athens (BRFAA) computer cluster that consists of 24 nodes of 128 GB RAM each. Each node consists of 2 Intel ${ }^{\circledR}$ Xeon ${ }^{\circledR}$ Silver 4116 processors with 12 cores per processor and 2 threads per core (i.e. 48 CPUs per node). Additionally, Homo sapiens assembly by Wengan was performed on an Aristotle University of Thessaloniki (AUTh) computational system on a single node which consists of 4 AMD Opteron ${ }^{\mathrm{TM}}$ 6274 processors with 16 cores per processor and 1 thread per core (i.e., 64 CPUs) and 256 GB RAM.

The pipeline is divided into 3 parts: In the first stage of the current workflow (Fig. 1), different assemblers were used for the genome construction. In the second stage, the scaffolding, Hi-C data were combined with the initial assembly, in order to increase its continuity and accuracy. In the last stage, the final assembly was assessed and evaluated with the use of various tools.

Genome assembly. In order to assess the hybrid assembly strategy, the present study chose to evaluate two pipelines, MaSuRCA (version 3.3.5) (27,28) and Wengan (version 0.1) (29). MaSuRCA workflow offers three different assemblers, CABOG (30), SOAPdenovo (31) and Flye (32). The pipeline was tested using CABOG and Flye assemblers, which are designed for long-read assembly. Wengan pipeline is based on DiscovarDenovo assembler (33).

Canu (version 2.0) (34) is a long-read assembler, designed to use long high-noise single-molecule sequencing data, such as Nanopore and PacBio reads. Its workflow is based on the Celera assembler (35) which was used in the Human Genome Project to produce the first draft of the human genome. Hifiasm (version 0.13) (36) and HiCanu (Canu version 2.1.1) (37) are long-read assemblers exclusively for $\mathrm{HiFi}$ reads. The main difference between HiFi assemblers and the ones mentioned previously, is that Hifiasm and HiCanu produce phased assemblies. A phased assembly is a haplotype-resolved assembly, where high complexity regions, such as genes, will be separated into two different alleles $(36,38)$. HiCanu is a modified version of Canu, adapted to take advantage of the characteristics of $\mathrm{HiFi}$ reads. Hifiasm produces two different files for the primary and alternative assembly, whereas HiCanu combines the primary and the alternative assembly in the same FASTA file.

Scaffolding. In order to test the necessity of scaffolding, a scaffolder was used to improve the assembly continuity and completeness, as follows: $\mathrm{Hi}-\mathrm{C}$ data are mapped to the primary assembly by Arima mapping pipeline (39), to produce a BAM file which is consequently converted to a BED file. SALSA (version 2.2) (40) uses this BED file which contains the mapping information of $\mathrm{Hi}-\mathrm{C}$ reads on the assembly, to scaffold the primary assembly.

Quality control metrics. For the quality control of the assemblies produced, different evaluation tools were used. These tools produce and present the qualitative and quantitative 
Table I. ENA accessions and T2T links of primary sequencing data.

\begin{tabular}{|c|c|c|c|c|c|}
\hline Organism & $\begin{array}{l}\text { Genome } \\
\text { size } \\
(\mathrm{Mbp})\end{array}$ & $\begin{array}{l}\text { Illumina paired-end } \\
\text { sequencing } \\
\text { (coverage) }\end{array}$ & $\begin{array}{l}\text { Illumina Hi-C } \\
\text { sequencing } \\
\text { (coverage) }\end{array}$ & $\begin{array}{l}\text { Nanopore } \\
\text { reads } \\
\text { (coverage) }\end{array}$ & $\begin{array}{l}\mathrm{PacBio} / \mathrm{HiFi} \\
\text { reads } \\
\text { (coverage) }\end{array}$ \\
\hline Drosophila virilis & 169 & $\begin{array}{l}\text { SRR1536175 } \\
(108 x)\end{array}$ & $\begin{array}{l}\text { SRR7029394 } \\
(67 x)\end{array}$ & $\begin{array}{l}\text { SRR7167958 } \\
(50 x)\end{array}$ & \\
\hline Drosophila melanogaster & 140 & & & & $\begin{array}{l}\text { SRR9969842 (37x), } \\
\text { SRR10238607 } \\
\text { (subsampled to 92x) }\end{array}$ \\
\hline Homo sapiens & 3,200 & $\begin{array}{l}\text { SRR3189741 } \\
\text { SRR3189742 } \\
\text { (Combined and } \\
\text { subsampled } \\
\text { to 34x) }\end{array}$ & $\begin{array}{l}\text { https://github.com/ } \\
\text { nanopore-wgs- } \\
\text { consortium/ } \\
\text { CHM13\#hi-c-data } \\
(40 x)\end{array}$ & $\begin{array}{l}\text { https://github.com/ } \\
\text { nanopore-wgs- } \\
\text { consortium/ } \\
\text { CHM13\#oxford- } \\
\text { nanopore-data } \\
\text { (Subsampled } \\
\text { to } 30 \mathrm{x} \text { ) }\end{array}$ & $\begin{array}{l}\text { SRR11292120 } \\
\text { SRR11292121 } \\
\text { SRR11292122 } \\
\text { SRR11292123 } \\
\text { (Combined and } \\
\text { subsampled } \\
\text { to 16x) }\end{array}$ \\
\hline
\end{tabular}

In some cases, where more than one FASTQ files was used, the files were combined and randomly subsampled to lower coverages. Mbp, Megabase pairs.

Table II. Reference genomes used for the evaluation of the assemblies.

\begin{tabular}{ll}
\hline Organisms & \multicolumn{1}{c}{ Reference genomes } \\
\hline Drosophila virilis & GCA_007989325.1_vir160_genomic.fna \\
& https://ftp.ncbi.nlm.nih.gov/genomes/all/GCA/007/989/325/GCA_007989325.1_vir160/ \\
Drosophila melanogaster & GCA_002300595.1_Dmel_A4_1.0_genomic.fna \\
& https://ftp.ncbi.nlm.nih.gov/genomes/all/GCA/002/300/595/GCA_002300595.1_Dmel_A4_1.0/ \\
Homo sapiens & chm13.draft_v1.0.fasta \\
& https://s3.amazonaws.com/nanopore-human-wgs/chm13/assemblies/chm13.draft_v1.0.fasta.gz \\
\hline
\end{tabular}

characteristics of the assemblies in a comprehensible way. QUAST (version 5.0.2) (41), a genome assembly evaluation tool, produces various metrics for our assemblies, using a reference genome (Table II). The standard assembly statistics include the calculation of N50/NG50 and L50/LG50 values (42), as follows: N50 (or NG50) is the size of the contig, where at least $50 \%$ of the genome assembly size (or the reference genome size), is contained in contigs of equal or larger size than this contig. Higher N50/NG50 values signify more contiguous assemblies. L50 (or LG50) is the smallest number of contigs whose length sum makes up for at least $50 \%$ of the genome assembly length (or reference genome length). Lower L50/LG50 values signify more contiguous assemblies. Furthermore, QUAST makes use of BUSCO (Quast version 5.0.2) (43), to assess genome assembly and annotation completeness, based on evolutionarily-informed expectations of gene content of near-universal single-copy orthologs.

Genome consistency plots. JupiterPlot (version 1.0) (44) is a workflow that uses Circos (45) to generate a genome assembly consistency plot between a reference genome and a genome assembly. The chromosomes of the reference genome are represented as coloured arcs on the left half circle of the plot, whereas the contigs/scaffolds of the assembled genome are represented as outlined white arcs on the right half circle. The number and size of white arcs is indicative of the genome contiguity. JupiterPlot represents synteny between the reference and the assembled genome, indicating corresponding contiguous regions as ribbons whose width is proportional to their sequence length. In this manner, assembly errors and chromosomal misassemblies can be visually identified: A ribbon in twisted position represents an inversion, a ribbon which crosses over other ribbons represents a translocation, a lack of a ribbon connecting a region of the reference genome represents a deletion and the overlap of two ribbons connecting the same reference genome region represents a duplication. Although in other cases these misassemblies may represent genuine chromosomal aberrations, in our case they represent assembly errors due to low sequence complexity of repetitive regions such as centromeres, telomeres, etc., low sequencing coverage and weaknesses of each assembly algorithm.

\section{Results}

Drosophila genome assemblies. Primary (unscaffolded) MaSuRCA (CABOG or Flye) hybrid assemblies are by far the 
Table III. Metrics of Drosophila assemblies.

\begin{tabular}{|c|c|c|c|c|c|c|}
\hline Assemblers & $\begin{array}{l}\text { Contigs/ } \\
\text { scaffolds }\end{array}$ & $\begin{array}{l}\text { Genome assembly } \\
\text { size (bp) }\end{array}$ & N50 & NG50 & L50 & LG50 \\
\hline MaSuRCA (CABOG) & 1,016 & $167,374,624$ & 366,859 & 359,873 & 127 & 131 \\
\hline MaSuRCA (CABOG)/SALSA (Arima) & 532 & $167,617,624$ & $3,400,369$ & $3,400,369$ & 15 & 15 \\
\hline MaSuRCA (Flye) & 689 & $163,000,738$ & 419,467 & 406,899 & 113 & 121 \\
\hline MaSuRCA (Flye)/SALSA (Arima) & 230 & $163,230,238$ & $5,261,864$ & $5,258,634$ & 9 & 10 \\
\hline Wengan & 329 & $153,989,049$ & $3,232,846$ & $3,013,042$ & 13 & 16 \\
\hline Wengan/SALSA (Arima) & 229 & $154,046,842$ & $21,036,706$ & $16,232,289$ & 3 & 4 \\
\hline Canu & 425 & $169,315,961$ & $4,435,749$ & $4,435,749$ & 10 & 10 \\
\hline Canu/SALSA (Arima) & 488 & $176,029,265$ & $25,182,285$ & $25,182,285$ & 4 & 4 \\
\hline $\begin{array}{l}\text { Hifiasm } \\
\text { Insert size: } 11 \mathrm{Kbp} \\
\text { Coverage: } 37 \mathrm{x}\end{array}$ & 314 & $149,971,598$ & $23,693,975$ & $23,693,975$ & 3 & 3 \\
\hline $\begin{array}{l}\text { Insert size: } 24 \mathrm{Kbp} \\
\text { Coverage: } 40 \mathrm{x}\end{array}$ & 149 & $164,010,561$ & $21,707,601$ & $24,110,342$ & 4 & 3 \\
\hline $\begin{array}{l}\text { Insert size: } 24 \mathrm{Kbp} \\
\text { Coverage: } 92 \mathrm{x}\end{array}$ & 186 & $169,871,295$ & $23,943,049$ & $24,211,538$ & 4 & 3 \\
\hline $\begin{array}{l}\text { Hifiasm/SALSA } \\
\text { Insert size: } 11 \mathrm{Kbp} \\
\text { Coverage: } 37 \mathrm{x}\end{array}$ & 308 & $149,976,098$ & $23,693,975$ & $23,693,975$ & 3 & 3 \\
\hline $\begin{array}{l}\text { Insert size: } 24 \mathrm{Kbp} \\
\text { Coverage: } 40 \mathrm{x}\end{array}$ & 141 & $164,015,561$ & $24,110,342$ & $24,620,248$ & 4 & 3 \\
\hline $\begin{array}{l}\text { Insert size: } 24 \mathrm{Kbp} \\
\text { Coverage: } 92 \mathrm{x}\end{array}$ & 183 & $169,876,757$ & $23,943,049$ & $24,211,538$ & 4 & 3 \\
\hline \multicolumn{7}{|l|}{ HiCanu } \\
\hline $\begin{array}{l}\text { Insert size: } 11 \mathrm{Kbp} \\
\text { Coverage: } 37 \mathrm{x}\end{array}$ & 1,792 & $295,986,869$ & $2,513,964$ & $6,791,534$ & 24 & 7 \\
\hline $\begin{array}{l}\text { Insert size: } 24 \mathrm{Kbp} \\
\text { Coverage: } 40 \mathrm{x}\end{array}$ & 1,024 & $322,211,690$ & $6,752,429$ & $17,694,921$ & 12 & 4 \\
\hline $\begin{array}{l}\text { Insert size: } 24 \mathrm{Kbp} \\
\text { Coverage: } 92 \mathrm{x}\end{array}$ & 1,269 & $337,795,659$ & $11,255,983$ & $26,987,095$ & 8 & 2 \\
\hline \multicolumn{7}{|l|}{ HiCanu/SALSA } \\
\hline $\begin{array}{l}\text { Insert size: } 11 \mathrm{Kbp} \\
\text { Coverage: } 37 \mathrm{x}\end{array}$ & 1,747 & $296,025,369$ & $5,836,825$ & $10,646,076$ & 14 & 4 \\
\hline $\begin{array}{l}\text { Insert size: } 24 \mathrm{Kbp} \\
\text { Coverage: } 40 \mathrm{x}\end{array}$ & 1,023 & $322,224,690$ & $12,833,112$ & $30,402,815$ & 7 & 2 \\
\hline $\begin{array}{l}\text { Insert size: } 24 \mathrm{Kbp} \\
\text { Coverage: } 92 \mathrm{x}\end{array}$ & 1,281 & $337,778,159$ & $6,830,725$ & $16,844,691$ & 12 & 4 \\
\hline
\end{tabular}

Hybrid assemblies (MaSuRCA and Wengan) and long-read Nanopore assembly (Canu) were based on the Drosophila virilis genome (size: 169773245). HiFi PacBio assemblies (Hifiasm and HiCanu) were based on the Drosophila melanogaster genome (size: 145940863). Hifiasm and HiCanu assembles were performed using three combinations of insert data and coverage.

most fragmented of all Drosophila virilis assemblies, based on N50/NG50 and L50/LG50 values (Table III) and manual inspection of genome assembly consistency plots (Fig. 2). Canu, based exclusively on long Nanopore data, produced the most contiguous primary assembly. MaSuRCA/CABOG produced the most misassembled contigs, while Wengan hybrid assembler created the least misassembled ones. All but Canu assemblies present very high rates of preserved gene completeness, similar to the rates of the reference genomes (Table IV). The sizes of all Drosophila virilis primary assemblies are comparable to each other and very similar to that of the reference genome. Wengan is the fastest hybrid assembler and produced the Drosophila virilis genome 71 times faster than Canu, while the average CPU usage of Wengan is smaller than the rest of these assemblers (Table V). Hi-C-based scaffolding ameliorated the contiguity and it limited the misassemblies of all assemblies, 
Table IV. BUSCO values of Drosophila assemblies.

\begin{tabular}{|c|c|c|c|c|}
\hline Assemblers & $\begin{array}{l}\text { Completed and } \\
\text { single-copy } \\
\text { BUSCOs (S) }\end{array}$ & $\begin{array}{l}\text { Completed and } \\
\text { duplicated } \\
\text { BUSCOs (D) }\end{array}$ & $\begin{array}{l}\text { Fragmented } \\
\text { BUSCOs (F) }\end{array}$ & $\begin{array}{c}\text { Missing } \\
\text { BUSCOs (M) }\end{array}$ \\
\hline Drosophila virilis reference genome & $98.0 \%$ & $0.5 \%$ & $0.7 \%$ & $0.8 \%$ \\
\hline MaSuRCA (CABOG) & $96.1 \%$ & $1.5 \%$ & $0.8 \%$ & $1.6 \%$ \\
\hline MaSuRCA (CABOG)/SALSA (Arima) & $96.1 \%$ & $1.4 \%$ & $0.8 \%$ & $1.7 \%$ \\
\hline MaSuRCA (Flye) & $98.2 \%$ & $0.5 \%$ & $0.8 \%$ & $0.5 \%$ \\
\hline MaSuRCA (Flye)/SALSA (Arima) & $98.0 \%$ & $0.5 \%$ & $0.8 \%$ & $0.7 \%$ \\
\hline Wengan & $98.0 \%$ & $0.4 \%$ & $0.7 \%$ & $0.9 \%$ \\
\hline Wengan/SALSA (Arima) & $97.9 \%$ & $0.3 \%$ & $0.8 \%$ & $1.0 \%$ \\
\hline Canu & $62.7 \%$ & $0.2 \%$ & $21.3 \%$ & $15.8 \%$ \\
\hline Canu/SALSA (Arima) & $64.0 \%$ & $0.3 \%$ & $20.7 \%$ & $15.0 \%$ \\
\hline Drosophila melanogaster reference genome & $97.9 \%$ & $0.7 \%$ & $0.9 \%$ & $0.5 \%$ \\
\hline \multicolumn{4}{|l|}{ Hifiasmx } & \\
\hline $\begin{array}{l}\text { Coverage: } 37 \mathrm{x} \\
\text { Insert size: } 24 \mathrm{Kbp}\end{array}$ & $98.1 \%$ & $0.6 \%$ & $0.7 \%$ & $0.6 \%$ \\
\hline $\begin{array}{l}\text { Coverage: } 40 \mathrm{x} \\
\text { Insert size: } 24 \mathrm{Kbp}\end{array}$ & $98.2 \%$ & $0.4 \%$ & $0.7 \%$ & $0.7 \%$ \\
\hline Coverage: 90x & $98.1 \%$ & $0.5 \%$ & $0.7 \%$ & $0.7 \%$ \\
\hline \multicolumn{4}{|l|}{ Hifiasm/SALSA } & \\
\hline $\begin{array}{l}\text { Coverage: } 37 \mathrm{x} \\
\text { Insert size: } 24 \mathrm{Kbp}\end{array}$ & $98.1 \%$ & $0.6 \%$ & $0.7 \%$ & $0.6 \%$ \\
\hline $\begin{array}{l}\text { Coverage: } 40 \mathrm{x} \\
\text { Insert size: } 24 \mathrm{Kbp}\end{array}$ & $98.2 \%$ & $0.4 \%$ & $0.7 \%$ & $0.7 \%$ \\
\hline Coverage: $90 \mathrm{x}$ & $98.2 \%$ & $0.5 \%$ & $0.7 \%$ & $0.6 \%$ \\
\hline \multicolumn{4}{|l|}{ HiCanu } & \\
\hline $\begin{array}{l}\text { Coverage: } 37 \mathrm{x} \\
\text { Insert size: } 24 \mathrm{Kbp}\end{array}$ & $4.8 \%$ & $94.1 \%$ & $0.6 \%$ & $0.5 \%$ \\
\hline $\begin{array}{l}\text { Coverage: } 40 \mathrm{x} \\
\text { Insert size: } 24 \mathrm{Kbp}\end{array}$ & $3.8 \%$ & $95.2 \%$ & $0.5 \%$ & $0.5 \%$ \\
\hline Coverage: 90x & $3.2 \%$ & $95.5 \%$ & $0.7 \%$ & $0.6 \%$ \\
\hline $\begin{array}{l}\text { HiCanu/SALSA } \\
\text { Insert size: } 11 \mathrm{Kbp}\end{array}$ & & & & \\
\hline $\begin{array}{l}\text { Coverage: } 37 \mathrm{x} \\
\text { Insert size: } 24 \mathrm{Kbp}\end{array}$ & $42.3 \%$ & $56.7 \%$ & $0.5 \%$ & $0.5 \%$ \\
\hline $\begin{array}{l}\text { Coverage: } 40 \mathrm{x} \\
\text { Insert size: } 24 \mathrm{Kbp}\end{array}$ & $37.3 \%$ & $61.6 \%$ & $0.5 \%$ & $0.6 \%$ \\
\hline Coverage: $90 \mathrm{x}$ & $39.0 \%$ & $59.9 \%$ & $0.5 \%$ & $0.6 \%$ \\
\hline
\end{tabular}

but it did not improve the gene completeness and it did not alter the final assembly size.

In Drosophila melanogaster primary assemblies, Hifiasm outperformed HiCanu, producing less fragmented and misassembled contigs (Figs. 3 and 4). As HiCanu produces phased assemblies, the vast majority of single-copy genes appeared as completed and duplicated in BUSCO analysis (Table IV). Nevertheless, the sum of completed single and duplicated
BUSCOs in Hifiasm and HiCanu was practically identical to that of the reference genome. While using the $11 \mathrm{Kbp}$ insert size and 37x coverage data, Hifiasm produced Drosophila melanogaster genome faster than HiCanu. However, as the coverage was increased, the assembly time of Hifiasm increased more rapidly than that of HiCanu: The assembly time of Hifiasm and HiCanu using $24 \mathrm{Kbp}$ insert size and 40x coverage data was approximately the same, while HiCanu 


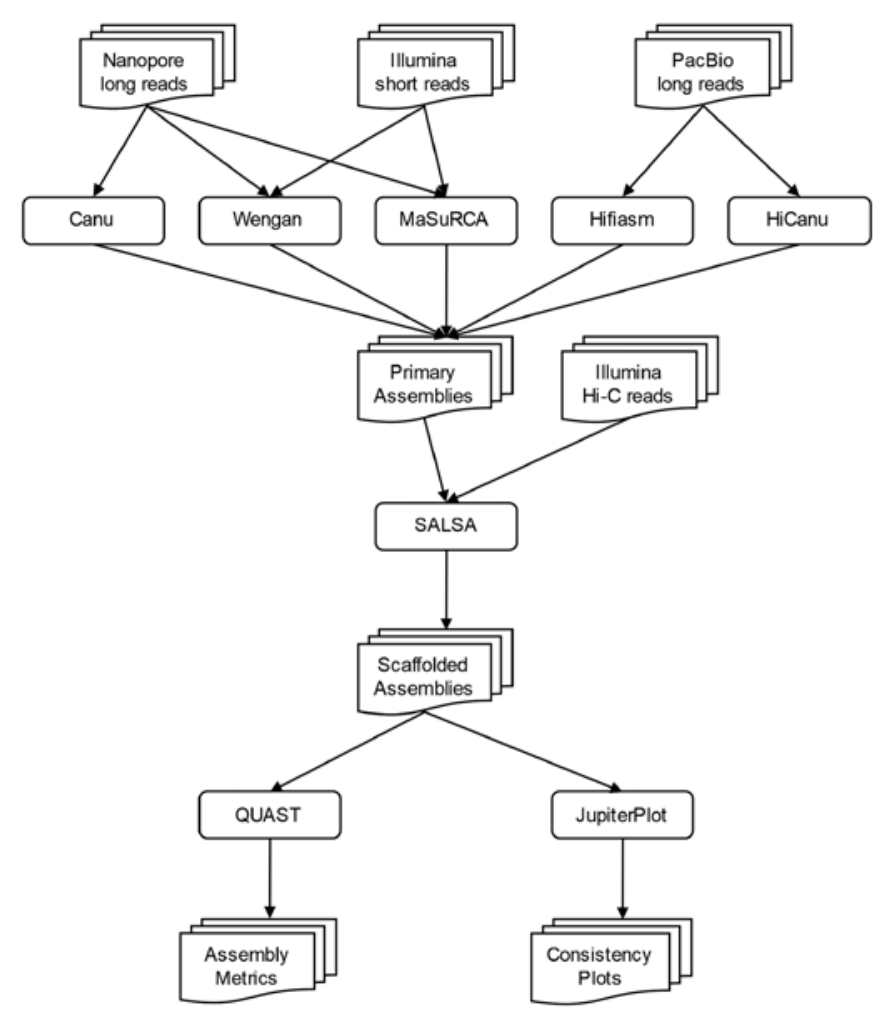

Figure 1. Pipeline stages and tools used in each step of the workflow.

was $12 \mathrm{x}$ faster than Hifiasm, when $24 \mathrm{Kbp}$ insert size and $92 x$ coverage was used. The average CPU usage of HiCanu was also smaller than that of Hifiasm (Table V). SALSA scaffolding based on Hi-C data, slightly improved Hifiasm assemblies, while it ameliorated the contiguity of HiCanu ones. It also slightly limited the misassemblies of HiCanu outputs. It did not influence the gene completeness of any assembly. Insert size (11 and $24 \mathrm{Kbp}$ ) and coverage (37x, 40x and 92x) did not influence the outcome of Hifiasm; however, a small deterioration in assembly contiguity at the $92 x$ coverage was noted. On the other hand, a higher insert size and coverage improved HiCanu performance.

Overall, Hifiasm performed most effectively in the primary assembly of Drosophila melanogaster genome (which is comparable to that of Drosophila virilis), in terms of genome contiguity, accuracy and completeness. At $37 \mathrm{x}$ and 40x coverages, Hifiasm was also the fastest assembler; however, the CPU usage of Wengan and HiCanu was half of that of Hifiasm. The combination of Hi-C data had a minimal effect on the improvement of Hifiasm assembly. Among hybrid assemblers, Wengan performed best when combined with SALSA.

Homo sapiens genome assemblies. The human genome is much more complex than that of Drosophila; thus, its assembly is a more demanding task which requires much more computational resources. MaSuRCA and Wengan hybrid assemblers and Canu long-read assembler, were not able to complete the assembly of the human genome, even in half of the original Illumina and Nanopore coverage, on the BRFAA cluster with 128 GB RAM. Wengan, though, was able to produce a human genome assembly on AUTh
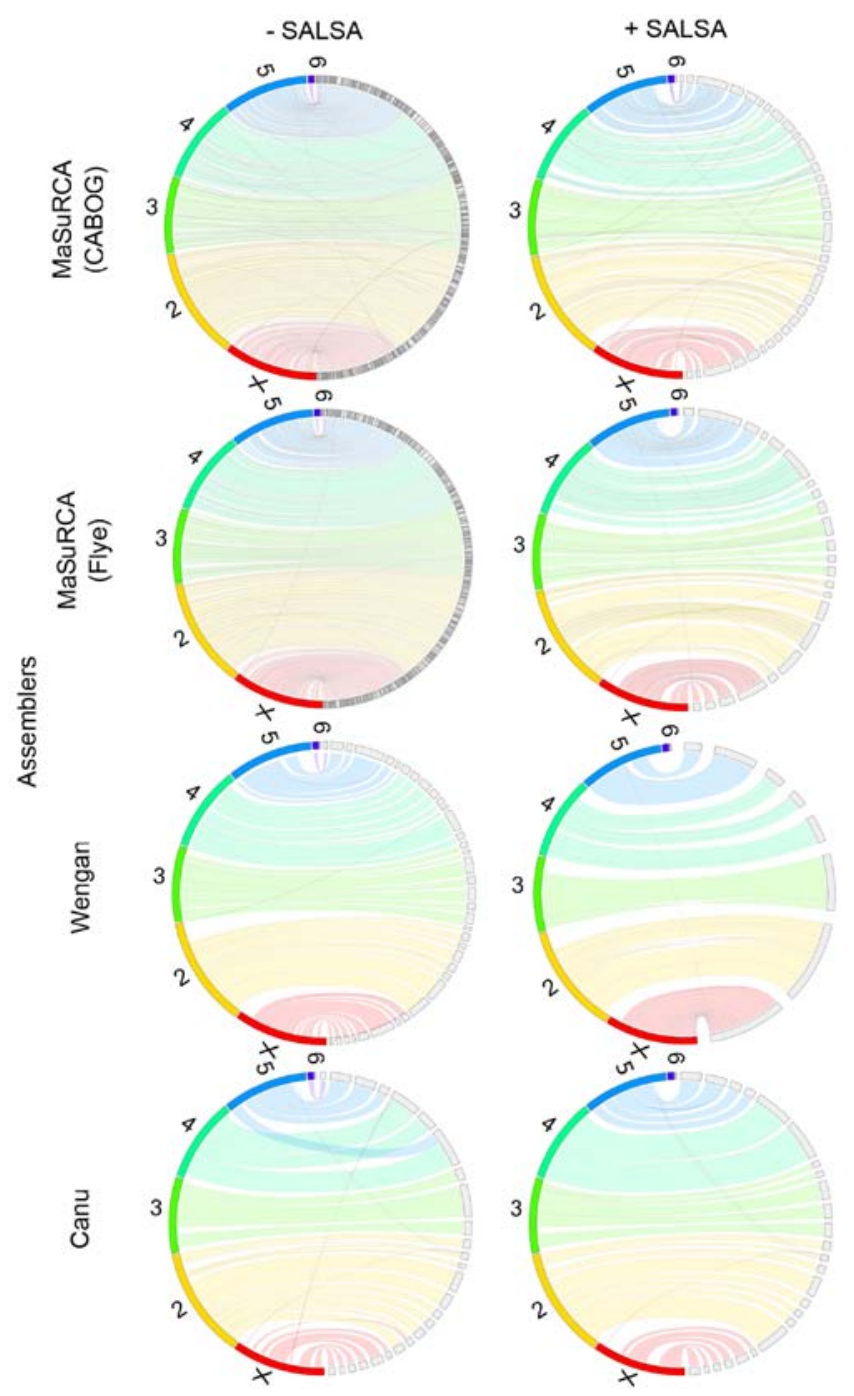

Figure 2. Drosophila virilis assemblies comparison. Hybrid assemblers, MaSuRCA (CABOG and Flye) and Wengan, used Illumina short reads and Nanopore long reads for the assembly, while Canu, a long read assembler utilised Nanopore long reads for the same purpose. SALSA improved contiguity in all assemblies.

computational system with 256 GB RAM, when FASTQ files were subsampled by half (Fig. 5). The incorporation of $\mathrm{Hi}-\mathrm{C}$ data improved the genome continuity and completeness, while reducing misassemblies (Table VI).

Hifiasm was unable to assemble the human genome on the BRFAA cluster when the original 30x coverage of $\mathrm{HiFi}$ data was used. Nevertheless, it succeeded to produce a notable assembly on the same computational system with subsampled data (16x coverage), in contrast to HiCanu, which failed to run because of low memory resources, even with the subsampled data. Hifiasm failed to produce a contig for chromosome 22. SALSA improved the contiguity, accuracy and completeness of Hifiasm assembly (Table VI). The longest chromosomes of the genome are well assembled, however, four of the smallest autosomal chromosomes (chr 16, 19, 21, 22) are missing (Fig. 5).

Hifiasm outperformed HiCanu, Canu, Wengan and MaSuRCA, as it managed to run in low resources and low coverage, producing superior primary and scaffolded assemblies to those of Wengan. 
Table V. Assembly time and CPU usage comparison.

\begin{tabular}{|c|c|c|c|c|}
\hline Organism & Assemblers & CPU time (sec) & CPU usage & $\begin{array}{l}\text { Elapsed (wall clock) } \\
\text { time (h:mm:ss) }\end{array}$ \\
\hline \multirow[t]{4}{*}{ Drosophila virilis } & MaSuRCA (CABOG) & $1,638,637.72$ & $3,954 \%$ & $11: 30: 39$ \\
\hline & MaSuRCA (Flye) & $1,344,633.10$ & $3,961 \%$ & $9: 25: 44$ \\
\hline & Canu & $993,441,898$ & $3,532 \%$ & $78: 07: 27$ \\
\hline & Wengan & $198,241.94$ & $2,831 \%$ & $1: 56: 42$ \\
\hline \multirow[t]{12}{*}{ Drosophila melanogaster } & Hifiasm & & & \\
\hline & Insert size: $11 \mathrm{Kbp}$ & & & \\
\hline & Coverage: $37 \mathrm{x}$ & $163,816.92$ & $4,098 \%$ & $1: 06: 37$ \\
\hline & Insert size: $24 \mathrm{Kbp}$ & & & \\
\hline & $\begin{array}{l}\text { Coverage: } 40 \mathrm{x} \\
\text { Insert size: } 24 \mathrm{Kbp}\end{array}$ & $215,855.05$ & $4,287 \%$ & $1: 23: 54$ \\
\hline & Coverage: $90 x$ & $4,271,030.94$ & $4,313 \%$ & $25: 40: 58$ \\
\hline & $\begin{array}{l}\text { HiCanu } \\
\text { Insert size: } 11 \mathrm{Kbp}\end{array}$ & & & \\
\hline & Coverage: $37 x$ & $85,224.85$ & $1,752 \%$ & $1: 21: 03$ \\
\hline & Insert size: $24 \mathrm{Kbp}$ & & & \\
\hline & Coverage: $40 x$ & $107,146.65$ & $2,235 \%$ & $1: 19: 53$ \\
\hline & Insert size: $24 \mathrm{Kbp}$ & & & \\
\hline & Coverage: $90 x$ & $176,649.77$ & $1,646 \%$ & $2: 58: 46$ \\
\hline Homo sapiens & Hifiasm & $1,272,271.15$ & $4,113 \%$ & $8: 35: 29$ \\
\hline
\end{tabular}

Table VI. Homo sapiens assembly metrics.

\begin{tabular}{|c|c|c|c|c|c|c|}
\hline Assemblers & Contigs/scaffolds & $\begin{array}{c}\text { Genome assembly } \\
\text { size (bp) }\end{array}$ & N50 & NG50 & L50 & LG50 \\
\hline Reference & 24 & $3,056,916,522$ & $154,259,625$ & & 8 & \\
\hline Wengan & 2,000 & $2,845,883,522$ & $39,733,923$ & $36,783,291$ & 23 & 26 \\
\hline Wengan/SALSA (Arima) & 1,689 & $2,845,883,522$ & $59,573,195$ & $56,310,190$ & 15 & 17 \\
\hline Hifiasm & 498 & $3,045,796,332$ & $45,256,540$ & $45,256,540$ & 20 & 20 \\
\hline Hifiasm/SALSA (Arima) & 431 & $3,045,840,332$ & $61,206,687$ & $61,206,687$ & 15 & 15 \\
\hline
\end{tabular}

\section{Discussion}

The use of a reference genome in the study of medical genetics, with the help of novel tools and methods, can help the identification of novel drug-sequence variant interactions (46) and the identification of variants which may be related to mutations with a genetic base of a variety of genetic diseases, such as cancer (47) and produce further analysis (48). By studying these variants, we are able to analyse the differences and the heterogeneity of different populations in order to understand their differences (49).

To propose an optimised de novo genome assembly workflow, in the present study, factors such as the maximum assembly contiguity, accuracy and completeness were taken into account, without ignoring other parameters crucial for the execution of the sequencing experiments and the production of the assemblies, such as financial, computational power and time limitations.

These findings suggest that the assembly exclusively based on long highly accurate PacBio Hifi reads outperforms
Illumina-Nanopore hybrid and Nanopore assembly. de novo genome assemblers which use $\mathrm{HiFi}$ reads, require lower amounts of data compared to other strategies. It has been reported that a 30x genome coverage, using $\mathrm{HiFi}$ data, is sufficient in order to produce high quality assemblies $(18,50)$. The present study revealed that even a 16x coverage of the human genome was adequate for that purpose. Thus, subsampling in Hifiasm assembly strategy allows the adaptation of sequencing data to the computational resources available as follows: Sequencing data with a coverage of no higher than $40 \mathrm{x}$ can be produced as the current findings and previous experience from other Hifiasm users (https://downloads.pacbcloud.com/public/dataset/redwood2020/hifiasm/v12/) suggest, and if the computational system fails to run, the data can be subsampled using the divide and conquer approach, until the computational resources are adequate for the analysis. However, if the subsampled data correspond to $<30 \mathrm{x}$ coverage, the final assembly can be deteriorated, as we notice on Homo sapiens assembly, where chromosome 22 is missing from the primary 

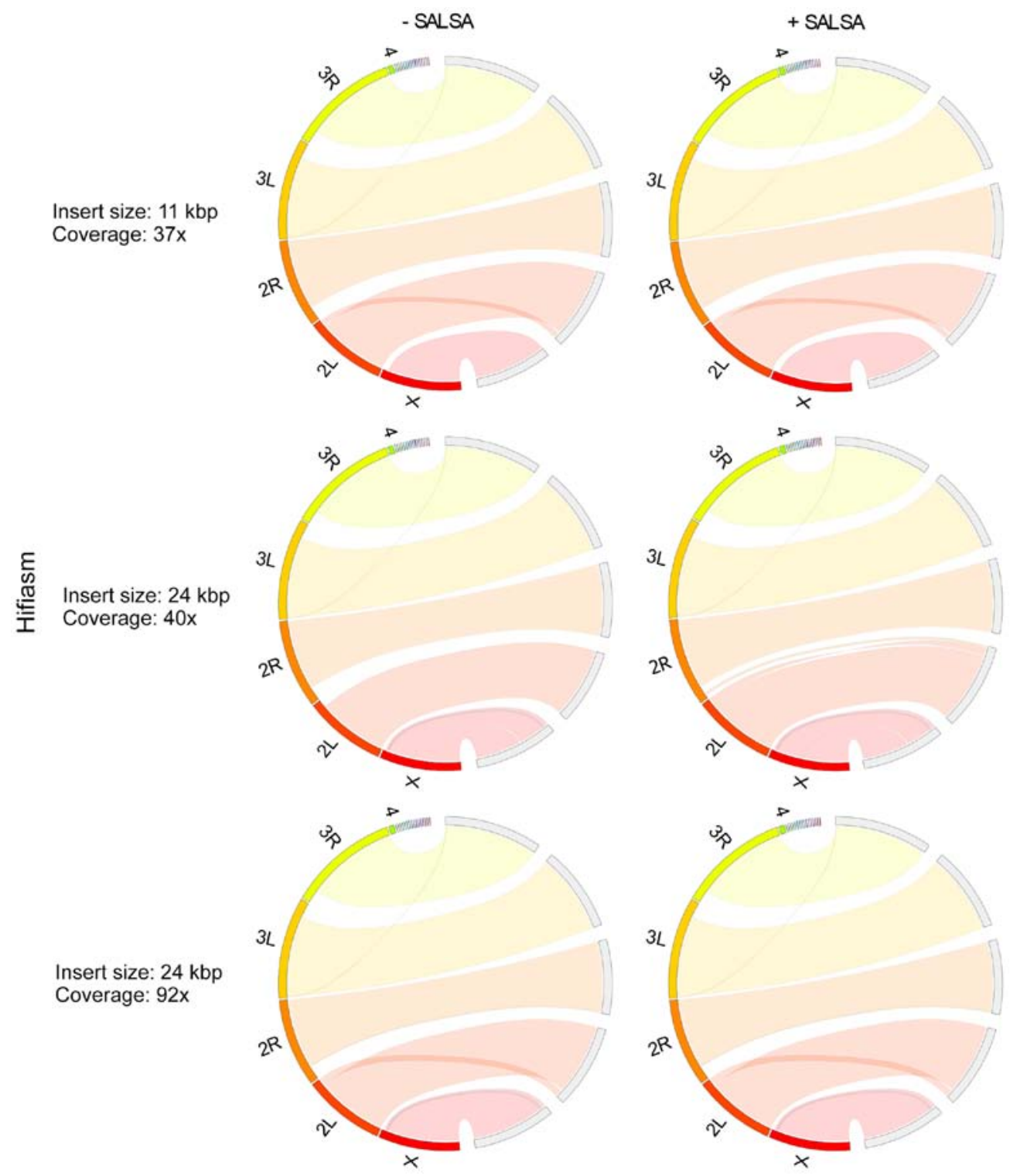

Figure 3. Drosophila melanogaster Hifiasm assemblies comparison. Hifiasm performed three different assemblies using PacBio Hifi long reads with different insert size (11 Kbp, $24 \mathrm{Kbp})$ and coverage (37x, 40x, 92x). A region in one of the two termini of chr 2L appears translocated in the assemblies produced by $11 \mathrm{Kbp}$ insert size with $37 \mathrm{x}$ coverage and $24 \mathrm{Kbp}$ insert size with $92 \mathrm{x}$ coverage. The same region appears deleted in the assembly produced by $24 \mathrm{Kbp}$ insert size with 40x coverage prior to SALSA scaffolding and inverted in the same assembly with SALSA scaffolding.

assembly and chromosomes 16, 19, 21 and 22 from the final assembly, after the scaffolding and correction process. On the other hand, it has been reported that a hybrid assembly would need 50x Illumina short-read coverage and 30x Nanopore long-read coverage of the genome $(15,51,52)$. In the case of the human genome, notable results with a 34x Illumina and 30x Nanopore coverage were able to be produced. Therefore, the volume of data used for HiFi assemblies is much smaller. As the volume of data decreases, so do the computational requirements for CPU power and particularly memory. In addition, the use of highly accurate long reads, bypasses several computationally demanding, time consuming steps of the assembly workflow.

In hybrid assembly strategy, Wengan performed most effectively in terms of accuracy and speed. Wengan produced the most contiguous Drosophila virilis assemblies. Although no hybrid assembler produced a human genome assembly in BRFAA cluster, Wengan was the only assembler that managed to construct a primary assembly in AUTh computational system.

The assembler we recommend for HiFi reads is Hifiasm, as it outperformed HiCanu in a small genome and it succeeded to produce a notable assembly of a large genome whereas HiCanu failed to run. Hifiasm performed equally well in respect of insert size and coverage, while HiCanu output improves with the increase in insert size and coverage. We recommend the use of Hifiasm or HiCanu assemblers, depending on the available computational resources as well as the organism's genome size and complexity. Hifiasm produced the most contiguous assemblies and its assembly strategy is highly efficient in terms of computational power and time on a single node of the cluster. For this reason, Hifiasm is also used by the Human Pangenome Project (https://humanpangenome.org/). On the other hand, HiCanu gives the possibility to run the assembly on grid when using a computational cluster. Distributing the tasks on different nodes allows the use of more computational 

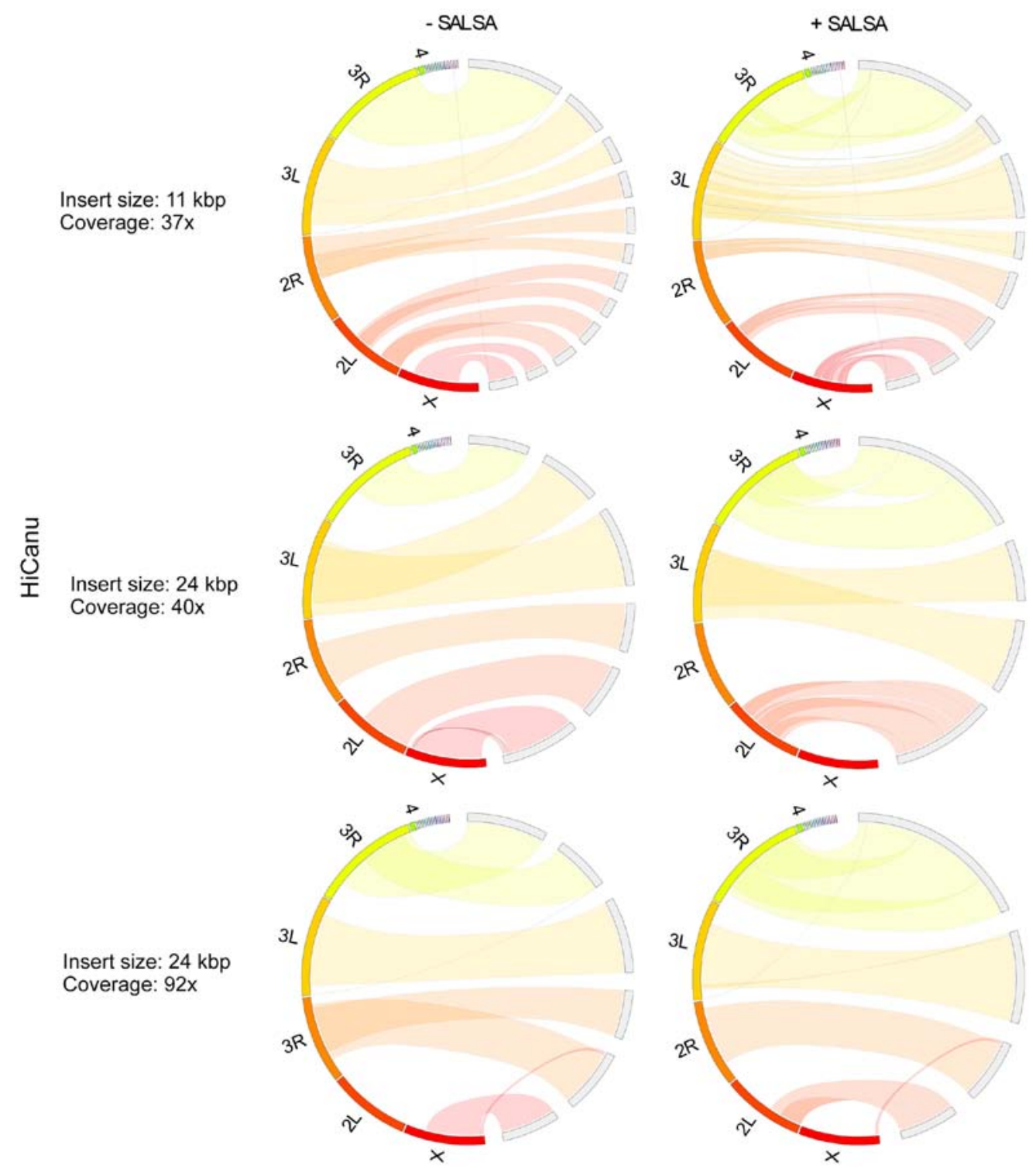

Figure 4. Drosophila melanogaster HiCanu assemblies comparison. HiCanu performed three different assemblies using PacBio Hifi long reads with different insert size (11 Kbp, $24 \mathrm{Kbp})$ and coverage (37x, 40x, 92x). Deletions of major regions or entire chromosomes can be found in all assemblies. Apparent duplications as of major parts of chr $3 \mathrm{~L}$ in the assemblies produced by $24 \mathrm{Kbp}$ insert size with $40 \mathrm{x}$ coverage are the results of phasing.

resources than running on a central resource and jobs can be executed in parallel speeding performance. Although running on grid, HiCanu was unable to produce a human genome assembly, as the main bottleneck of all assemblers is RAM size. Finally, by following PacBio HiFi assembly strategy for small genomes, we utilise only one sample preparation and one sequencing technology, in contrast to the Illumina/Nanopore hybrid strategy where we need to make three sample preparations (Illumina, Nanopore and Hi-C) and utilise two sequencing technologies (Illumina sequencing for short genomic and $\mathrm{Hi}-\mathrm{C}$ reads and Nanopore for long genomic reads). For larger genomes, similar to the human one, PacBio $\mathrm{HiFi}$ assembly strategy relies on two sample preparations and two sequencing technologies (Illumina sequencing for short $\mathrm{Hi}-\mathrm{C}$ reads and $\mathrm{PacBio}$ long genomic reads).

Our analysis suggests that the use of additional information for scaffolding is not necessary in small genomes (such as insect genomes); however, it offers a noticeable improvement in larger and more complex genomes (such as the human genome and higher plant genomes). The computational resources required for scaffolding, even for the most complex genomes, are far less than those for the assembly step. Ideally, the use of multiple types of data, seems to exploit different genome features. The successive use of 10x (https://www.10xgenomics.com/) $(53,54)$, Bionano (https://bionanogenomics.com/) (55) and Hi-C data will generate the most accurate scaffolds $(25,56)$. Although the use of 10x and Bionano data is not imperative, Hi-C sequencing reads are highly recommended for complex genomes, in order to increase the continuity of the assembly, while improving the accuracy by reducing major misassemblies and translocations.

The development of sequencing technologies led to a great reduction on sequencing cost. The purchase of a sequencer is no longer compulsory for genome assembly projects, as different institutes provide a variety of sequencing services at affordable, by many labs, prices. Each of PacBio, Illumina and Nanopore, offers a network of certified sequencing service providers. Some of these providers are certified for more than one of those sequencing technologies. Moreover, the 

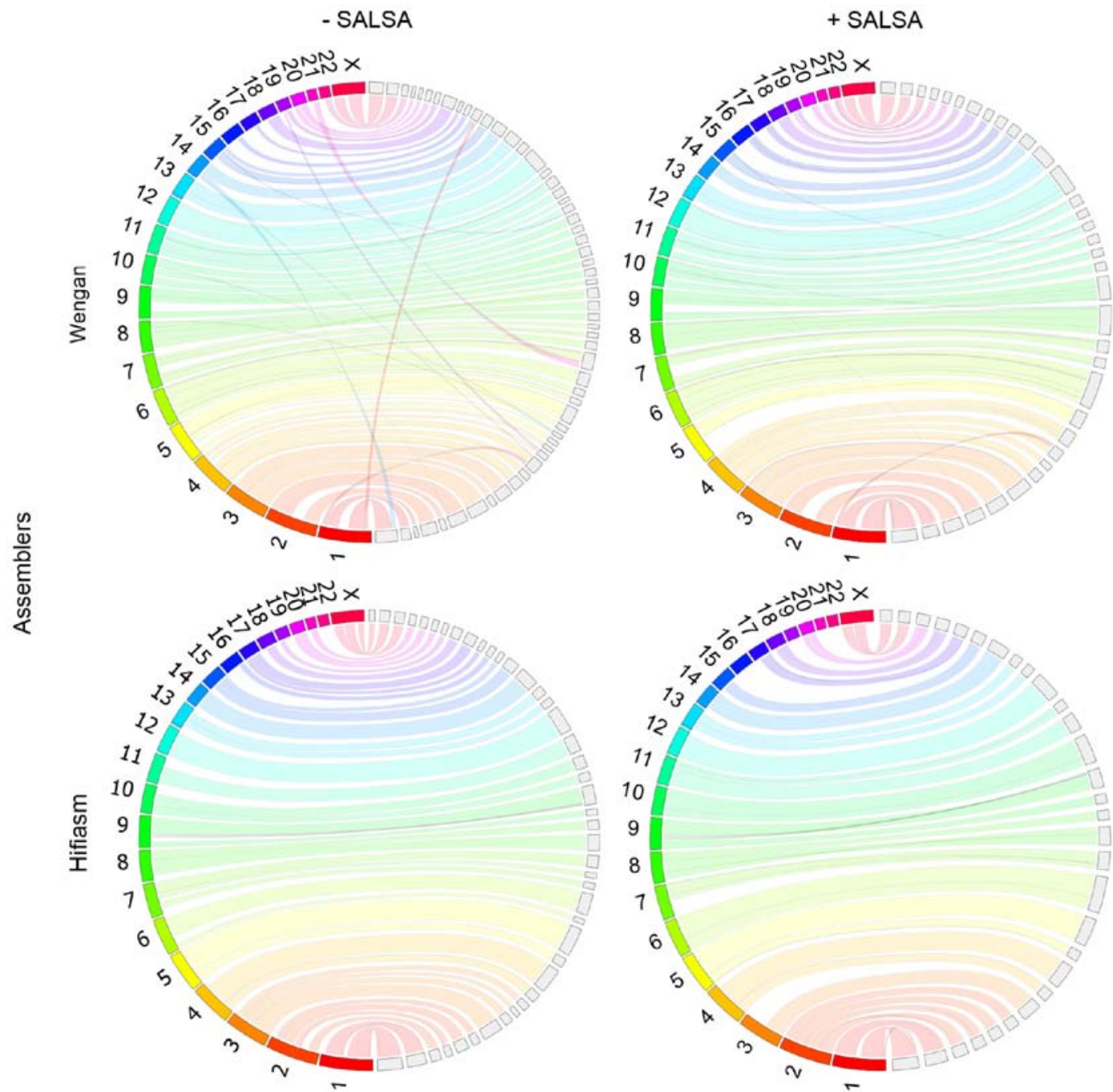

Figure 5. Homo sapiens assemblies comparison. Wengan hybrid assembler used 34x Illumina short reads and 30x Nanopore long reads for the assembly, while Hifiasm used 16x PacBio Hifi long reads.

purchase of a computational cluster is no longer necessary, as bioinformatics infrastructures, such as ELIXIR (57), can offer researchers the computational recourses necessary for the accomplishment of demanding tasks, such as a de novo genome assembly.

The major bottlenecks in genome assembly projects were the computationally demanding assembly algorithms and the large cost of sequencing. The development of new assembly algorithms, which require much less computational power and memory, is the result of major improvements in long-read accuracy by PacBio. The future of genomics relies on long-reads in order to resolve low complexity regions of the genomes and perform telomere-to-telomere assemblies. Alongside to the advances of read accuracy, third generation sequencing led to the reduction of sequencing cost. Furthermore, the increase of genomic data availability in public databases (58), such as Sequence Read Archive (SRA) (59), allows researchers to find and use a variety of raw sequencing data from the same species of interest, already produced by others, for the primary assembly and/or the scaffolding process. Finally, it is important to note that all assembly algorithms and methods we utilised during this work, are being constantly updated in order to improve in terms of performance and computational efficiency, allowing even the reanalysis of older data and the discovery of novel information. In addition, as basecallers are also constantly updated, reusing raw signal files (for example, fast5-formatted files in Nanopore) can produce more accurate reads.

In conclusion, continuous advancements in all fields mentioned above, lead towards the democratisation of de novo genome assembly projects, by enabling scientific laboratories with limited technical and financial resources to perform a great variety of genomic studies, without the need for expensive sequencing equipment and computational infrastructure.

\section{Acknowledgements}

The analyses of this work have been performed using the computing cluster of the Greek Genome Centre of the Biomedical Research Foundation, Academy of Athens and the Aristotle University of Thessaloniki (AUTh) High Performance Computing Infrastructure and Resources.

\section{Funding}

No funding was received. 


\section{Availability of data and materials}

The datasets used and/or analyzed during the current study are available from the corresponding author on reasonable request.

\section{Authors' contributions}

MG and KK analysed and interpreted the data. IM conceived and coordinated the current study. DAS was also involved in the conception of the study. MG and IM assessed the authenticity of all the raw data to ensure its legitimacy. All authors contributed to the writing and revision of the work and approved the final manuscript.

\section{Ethics approval and consent to participate}

Not applicable.

\section{Patient consent for publication}

Not applicable.

\section{Competing interests}

DAS is the Editor-in-Chief for the journal, but had no personal involvement in the reviewing process, or any influence in terms of adjudicating on the final decision, for this article. The other authors declare that they have no competing interests.

\section{References}

1. Lander ES, Linton LM, Birren B, Nusbaum C, Zody MC, Baldwin J, Devon K, Dewar K, Doyle M, FitzHugh W, et al International Human Genome Sequencing Consortium: Initial sequencing and analysis of the human genome. Nature 409: 860-921, 2001.

2. Sanger F, Nicklen S and Coulson AR: DNA sequencing with chain-terminating inhibitors. Proc Natl Acad Sci USA 74 5463-5467, 1977.

3. Kent WJ and Haussler D: Assembly of the working draft of the human genome with GigAssembler. Genome Res 11: 1541-1548, 2001.

4. Shendure J, Balasubramanian S, Church GM, Gilbert W, Rogers J, Schloss JA and Waterston RH: DNA sequencing at 40: Past, present and future. Nature 550: 345-353, 2017.

5. Salzberg SL and Yorke JA: Beware of mis-assembled genomes. Bioinformatics 21: 4320-4321, 2005.

6. Chaisson MJ, Wilson RK and Eichler EE: Genetic variation and the de novo assembly of human genomes. Nat Rev Genet 16: 627-640, 2015

7. van Dijk EL, Jaszczyszyn Y, Naquin D and Thermes C: The Third Revolution in sequencing technology. Trends Genet 34 666-681, 2018

8. Kasianowicz JJ, Brandin E, Branton D and Deamer DW: Characterization of individual polynucleotide molecules using a membrane channel. Proc Natl Acad Sci USA 93: 13770-13773, 1996.

9. Haque F, Li J, Wu HC, Liang XJ and Guo P: Solid-state and biological nanopore for real-time sensing of single chemical and sequencing of DNA. Nano Today 8: 56-74, 2013.

10. Eid J, Fehr A, Gray J, Luong K, Lyle J, Otto G, Peluso P, Rank D, Baybayan P, Bettman B, et al: Real-time DNA sequencing from single polymerase molecules. Science 323: 133-138, 2009.

11. Aird D, Ross MG, Chen W-S, Danielsson M, Fennell T, Russ C, Jaffe DB, Nusbaum C and Gnirke A: Analyzing and minimizing PCR amplification bias in Illumina sequencing libraries. Genome Biol 12: R18, 2011.

12. Jain M, Koren S, Miga KH, Quick J, Rand AC, Sasani TA, Tyson JR, Beggs AD, Dilthey AT, Fiddes IT, et al: Nanopore sequencing and assembly of a human genome with ultra-long reads. Nat Biotechnol 36: 338-345, 2018.
13. Lin Y, Yuan J, Kolmogorov M, Shen MW, Chaisson M and Pevzner PA: Assembly of long error-prone reads using de Bruijn graphs. Proc Natl Acad Sci USA 113: E8396-E8405, 2016.

14. Tan MH, Austin CM, Hammer MP, Lee YP, Croft LJ and Gan HM: Finding Nemo: Hybrid assembly with Oxford Nanopore and Illumina reads greatly improves the clownfish (Amphiprion ocellaris) genome assembly. Gigascience 7: 1-6, 2018.

15. Nowak RM, Jastrzębski JP, Kuśmirek W, Sałamatin R, Rydzanicz M, Sobczyk-Kopcioł A, Sulima-Celińska A, Paukszto Ł, Makowczenko KG, Płoski R, et al: Hybrid de novo whole-genome assembly and annotation of the model tapeworm Hymenolepis diminuta. Sci Data 6: 302, 2019.

16. Korlach J and Turner SW: Zero-Mode Waveguides. In: Encyclopedia of Biophysics. Roberts GC (ed). Springer, Heidelberg, pp2793-2795, 2013.

17. Wenger AM, Peluso P, Rowell WJ, Chang PC, Hall RJ, Concepcion GT, Ebler J, Fungtammasan A, Kolesnikov A, Olson ND, et al: Accurate circular consensus long-read sequencing improves variant detection and assembly of a human genome. Nat Biotechnol 37: 1155-1162, 2019.

18. Silvestre-Ryan J and Holmes I: Pair consensus decoding improves accuracy of neural network basecallers for nanopore sequencing. Genome Biol 22: 38, 2021.

19. Ghurye J and Pop M: Modern technologies and algorithms for scaffolding assembled genomes. PLoS Comput Biol 15: e1006994, 2019.

20. Lieberman-Aiden E, van Berkum NL, Williams L, Imakaev M, Ragoczy T, Telling A, Amit I, Lajoie BR, Sabo PJ, Dorschner MO, et al: Comprehensive mapping of long-range interactions reveals folding principles of the human genome. Science 326: 289-293, 2009.

21. Auton A, Brooks LD, Durbin RM, Garrison EP, Kang HM, Korbel JO, Marchini JL, McCarthy S, McVean GA and Abecasis GR; 1000 Genomes Project Consortium: A global reference for human genetic variation. Nature 526: 68-74, 2015.

22. Koepfli KP, Paten B and O'Brien SJ; Genome 10K Community of Scientists: The Genome 10K Project: A way forward. Annu Rev Anim Biosci 3: 57-111, 2015.

23. ICGC/TCGA Pan-Cancer Analysis of Whole Genomes Consortium: Pan-cancer analysis of whole genomes. Nature 578: 82-93, 2020.

24. Logsdon GA, Vollger MR, Hsieh P, Mao Y, Liskovykh MA Koren S, Nurk S, Mercuri L, Dishuck PC and Rhie A: The structure, function, and evolution of a complete human chromosome 8. bioRxiv: Sep 8, 2020 (Epub ahead of print). https://doi. org/10.1101/2020.09.08.285395.

25. Miga KH, Koren S, Rhie A, Vollger MR, Gershman A Bzikadze A, Brooks S, Howe E, Porubsky D, Logsdon GA, et al: Telomere-to-telomere assembly of a complete human X chromosome. Nature 585: 79-84, 2020.

26. Amid C, Alako BTF, Balavenkataraman Kadhirvelu V, Burdett T, Burgin J, Fan J, Harrison PW, Holt S, Hussein A, Ivanov E, et al: The European nucleotide archive in 2019. Nucleic Acids Res 48: D70-D76, 2020.

27. Zimin AV, Marçais G, Puiu D, Roberts M, Salzberg SL and Yorke JA: The MaSuRCA genome assembler. Bioinformatics 29: 2669-2677, 2013.

28. Zimin AV, Puiu D, Luo MC, Zhu T, Koren S, Marçais G, Yorke JA Dvořák $\mathbf{J}$ and Salzberg SL: Hybrid assembly of the large and highly repetitive genome of Aegilops tauschii, a progenitor of bread wheat, with the MaSuRCA mega-reads algorithm. Genome Res 27: 787-792, 2017.

29. Di Genova A, Buena-Atienza E, Ossowski S and Sagot MF: Wengan: Efficient and high quality hybrid de novo assembly of human genomes. bioRxiv: Nov 25, 2019 (Epub ahead of print). doi: https://doi.org/10.1101/840447.

30. Miller JR, Delcher AL, Koren S, VenterE, Walenz BP, Brownley A, Johnson J, Li K, Mobarry C and Sutton G: Aggressive assembly of pyrosequencing reads with mates. Bioinformatics 24: 2818-2824, 2008.

31. Luo R, Liu B, Xie Y, Li Z, Huang W, Yuan J, He G, Chen Y, Pan Q, Liu Y, et al: SOAPdenovo2: An empirically improved memory-efficient short-read de novo assembler. Gigascience 1: 18, 2012.

32. Kolmogorov M, Yuan J, Lin Y and Pevzner PA: Assembly of long, error-prone reads using repeat graphs. Nat Biotechnol 37: 540-546, 2019.

33. Weisenfeld NI, Yin S, Sharpe T, Lau B, Hegarty R, Holmes L, Sogoloff B, Tabbaa D, Williams L, Russ C, et al: Comprehensive variation discovery in single human genomes. Nat Genet 46: 1350-1355, 2014 
34. Koren S, Walenz BP, Berlin K, Miller JR, Bergman NH and Phillippy AM: Canu: Scalable and accurate long-read assembly via adaptive k-mer weighting and repeat separation. Genome Res 27: 722-736, 2017.

35. Myers EW, Sutton GG, Delcher AL, Dew IM, Fasulo DP Flanigan MJ, Kravitz SA, Mobarry CM, Reinert KH, Remington KA, et al: A whole-genome assembly of Drosophila. Science 287: 2196-2204, 2000

36. Cheng H, Concepcion GT, Feng X, Zhang $\mathrm{H}$ and Li $\mathrm{H}$ : Haplotype-resolved de novo assembly with phased assembly graphs. arXiv: Aug 3, 2020 (Epub ahead of print).

37. Nurk $S$, Walenz BP, Rhie A, Vollger MR, Logsdon GA, Grothe R, Miga KH, Eichler EE, Phillippy AM and Koren S: HiCanu: Accurate assembly of segmental duplications, satellites, and allelic variants from high-fidelity long reads. Genome Res 30 : 1291-1305, 2020.

38. Chin C-S, Peluso P, Sedlazeck FJ, Nattestad M, Concepcion GT, Clum A, Dunn C, O'Malley R, Figueroa-Balderas R, Morales-Cruz A, et al: Phased diploid genome assembly with single-molecule real-time sequencing. Nat Methods 13: 1050-1054, 2016.

39. Arima Genomics, Inc.: Arima-HiC Mapping Pipeline. San Diego, 2019: GitHub; https://github.com/ArimaGenomics/ mapping_pipeline/blob/master/Arima_Mapping_UserGuide_ A160156_v02.pdf.

40. Ghurye J, Rhie A, Walenz BP, Schmitt A, Selvaraj S, Pop M, Phillippy AM and Koren S: Integrating Hi-C links with assembly graphs for chromosome-scale assembly. PLoS Comput Biol 15: e1007273, 2019.

41. Gurevich A, Saveliev V, Vyahhi N and Tesler G: QUAST: Quality assessment tool for genome assemblies. Bioinformatics 29: 1072-1075, 2013

42. Earl D, Bradnam K, St John J, Darling A, Lin D, Fass J, Yu HO, Buffalo V, Zerbino DR, Diekhans M, et al: Assemblathon 1: A competitive assessment of de novo short read assembly methods. Genome Res 21: 2224-2241, 2011.

43. Seppey M, Manni M and Zdobnov EM: BUSCO: Assessing genome assembly and annotation completeness. Methods Mol Biol 1962: 227-245, 2019.

44. Chu J: Jupiter Plot: A circos-based tool to visualize genome assembly consistency, 2018: GitHub; https://github.com/ JustinChu/JupiterPlot/find/master.

45. Krzywinski M, Schein J, Birol I, Connors J, Gascoyne R, Horsman D, Jones SJ and Marra MA: Circos: An information aesthetic for comparative genomics. Genome Res 19: 1639-1645, 2009.

46. Kyriakidis K, Charalampidou A, Natsiavas P, Vizirianakis IS and Malousi A: Linking exome sequencing data with drug response aberrations. Stud Health Technol Inform 264: 1845-1846, 2019.

47. Wei X, Ju X, Yi X, Zhu Q, Qu N, Liu T, Chen Y, Jiang H, Yang $\mathrm{G}$, Zhen $\mathrm{R}$, et al: Identification of sequence variants in genetic disease-causing genes using targeted next-generation sequencing. PLoS One 6: e29500, 2011.
48. Kanakoglou DS, Michalettou TD, Vasileiou C, Gioukakis E, Maneta D, Kyriakidis KV, Georgakilas AG and Michalopoulos I: Effects of high-dose ionizing radiation in human gene expression: A meta-analysis. Int J Mol Sci 21: 21, 2020.

49. DePristo MA, Banks E, Poplin R, Garimella KV, Maguire JR, Hartl C, Philippakis AA, del Angel G, Rivas MA, Hanna M, et al: A framework for variation discovery and genotyping using next-generation DNA sequencing data. Nat Genet 43: 491-498, 2011.

50. Vollger MR, Logsdon GA, Audano PA, Sulovari A, Porubsky D, Peluso P, Wenger AM, Concepcion GT, Kronenberg ZN, Munson KM, et al: Improved assembly and variant detection of a haploid human genome using single-molecule, high-fidelity long reads. Ann Hum Genet 84: 125-140, 2020.

51. Chen Z, Erickson DL and Meng J: Benchmarking hybrid assembly approaches for genomic analyses of bacterial pathogens using Illumina and Oxford Nanopore sequencing. BMC Genomics 21: 631,2020

52. Johnson LK, Sahasrabudhe R, Gill JA, Roach JL, Froenicke L, Brown CT and Whitehead A: Draft genome assemblies using sequencing reads from Oxford Nanopore Technology and Illumina platforms for four species of North American Fundulus killifish. Gigascience 9: 9, 2020.

53. Coombe L, Zhang J, Vandervalk BP, Chu J, Jackman SD, Birol I and Warren RL: ARKS: Chromosome-scale scaffolding of human genome drafts with linked read kmers. BMC Bioinformatics 19: 234, 2018.

54. Yeo S, Coombe L, Warren RL, Chu J and Birol I: ARCS: Scaffolding genome drafts with linked reads. Bioinformatics 34: 725-731, 2018.

55. Lam ET, Hastie A, Lin C, Ehrlich D, Das SK, Austin MD, Deshpande P, Cao H, Nagarajan N, Xiao M, et al: Genome mapping on nanochannel arrays for structural variation analysis and sequence assembly. Nat Biotechnol 30: 771-776, 2012.

56. Wallberg A, Bunikis I, Pettersson OV, Mosbech MB, Childers AK, Evans JD, Mikheyev AS, Robertson HM, Robinson GE and Webster MT: A hybrid de novo genome assembly of the honeybee, Apis mellifera, with chromosome-length scaffolds. BMC Genomics 20: 275, 2019.

57. Crosswell LC and Thornton JM: ELIXIR: A distributed infrastructure for European biological data. Trends Biotechnol 30: 241-242, 2012.

58. Kodama Y, Shumway M and Leinonen R; International Nucleotide Sequence Database Collaboration: The sequence read archive: Explosive growth of sequencing data. Nucleic Acids Res 40: D54-D56, 2012

59. Leinonen R, Sugawara H and Shumway M; International Nucleotide Sequence Database Collaboration: The sequence read archive. Nucleic Acids Res 39: D19-D21, 2011.

This work is licensed under a Creative Commons Attribution-NonCommercial-NoDerivatives 4.0 International (CC BY-NC-ND 4.0) License. 